\title{
20. COMMISSION DES POSITIONS ET DES MOUVEMENTS DES PETITES PLANETES, DES COMETES, ET DES SATELLITES
}

\author{
Président: M. A. O. Leuschner, Director of the Students' Observatory of the \\ University of California, Berkeley, Cal., U.S.A.
}

Vice-Président: M. B. Noumerov.

Membres: MM. Arend, Asplind, Banachiewicz, Boda, Brouwer, R. Carrasco, Comrie, Crawford, Crommelin, de la Villemarqué, Delporte, Eckert, Esclangon, Fayet, Giacobini, Guth, Hammond, Heinrich, Hinks, K. Hirayama, Spencer Jones, Kamienski, Kepiński, Kobold, Komendantov, Kopff, Lagrula, E. G. Martin, E. L. Martin, Maxwell, Michkovitch, Nechvíle, G. Neuymin, Perrine, Reinmuth, Schaumasse, Schorr, Silva, Slocum, Stracke, E. Strömgren, van Biesbroeck, Mlle Vinter Hansen, MM. Volta, Whipple, Witt, Wood.

In view of the considerable ground covered by the Commission at its Paris meetings and the fairly complete record of the activities of institutes and observatories, etc. published in the Minutes, it has not been deemed profitable by the President to call for further reports in advance of the Stockholm meeting. At the Paris meeting it was agreed that such reports be printed independently before each meeting of the Union and that reprints of or references to the published reports be sent to the President. It is hoped that all such reports if ready will be made available before the Stockholm meeting so that they may be summarized by the representatives in attendance or by the President and recorded in the Minutes. With reference to the pronouncement at the Paris meeting "that it is eminently desirable that more attention be given to the development of accurate general perturbations and mean elements on the basis of accurate osculating elements", the President has visited the Planeten-Institut at Frankfurt and the RechenInstitut at Berlin and has been in correspondence with the Leningrad Institute. From these sources particularly valuable material has been received. In this connection mention may be made that the Rechen-Institut printed a report entitled: "Ueber die rechnerische Behandlung der Bahnen der Kleinen Planeten", $A . N$. No. $6 \mathrm{I}_{58}$, and that beginning with Circular No. 1270 it has listed all planets for which general perturbations are available. To correlate all the material on general perturbations in a comprehensive form will require further time after the Stockholm meetings, but a general plan should be discussed at the meeting on the basis of suggestions to be made by the President. These suggestions will take cognizance of general theories and tables which have been published since the last meeting.

The very thorough discussions which took place at Paris on the various questions on the agenda happily have resulted in far greater uniformity of practice relating to the functions of the Commission. No suggestions have been received by the President from any member of the Commission for resolutions to be adopted or of new topics to be discussed. It has, however, been the experience of the Commission that during its sessions important matters were brought forth and it is hoped that this will again be the case at Stockholm. Reference to the Minutes of the Paris meeting, Transactions, I.A.U., volume 5, I935, pp. 309-26, will reveal a number of proposals which were held in abeyance for future discussions. These will be presented again.

With some of these proposals other Commissions are concerned and they have been placed on agenda by their Presidents for consideration at joint meetings. 
In this connection, particular reference should be made to the Preliminary Report of Commission 4. The President has requested the sub-committees on Solar Parallax and Periodic Comets to submit reports as part of the agenda of the Stockholm meeting.

It seems desirable that the Commission agree on a special plan of co-operation to secure suitable observations of such bodies as the recent Reinmuth Object (I937 UB) for determination of the distance by parallax methods and for the determination of as accurate elements as possible so as to prevent loss.

\section{Report of Sub-Committee on Solar Parallax}

I. Reference Stars. A catalogue of the definitive positions of the secondary reference stars for the I93I opposition of Eros has been published in Mem. R.A.S. 65, part iv, 1937. The typescript was prepared at Greenwich and the cost of reproduction by the replika process was defrayed by the Royal Astronomical Society. The catalogue was based on the measures of five series of plates, obtained at the Bergedorf, Leipzig, Greenwich, Lick and Cape Observatories. These plates were measured at the respective observatories, with the exception of the Cape series, which was measured at Greenwich. The R.A.'s and Dec.'s for I930 0 for 5623 secondary reference stars are given.

Corrections to the meridian positions of the primary reference stars, according to the Catalogue by Kopff, Nowacki and Gondolatsch (Ast. Nach. 24I, Nos. 578I-2; 244, Nos. 5852-3), derived from the photographic measures, are also given in the catalogue.

In order that all the positions of Eros may be on a uniform basis, it is important that all reductions of the observations of Eros obtained near the opposition of I93I should be based on the definitive positions of primary and secondary stars given in this catalogue. If other positions have been used for provisional reductions, it is hoped that the necessary corrections to reduce to the definitive basis may be determined and applied.

2. Measurement and Reduction of Observations of Eros. The present states of measurement and reduction of the photographic plates and of the reduction of micrometric observations are based on reports received from the co-operating observatories.

(a) Photographic Observations.

Greenwich. The measurement in both co-ordinates of the following series of plates has been completed:

(i) The Greenwich series of 160 plates with the 26 -inch refractor.

(ii) The Greenwich series of 204 plates with the I3-inch refractor.

(iii) The series of 52 plates obtained at the Radcliffe Observatory with the 24-inch refractor.

(iv) The series of 478 plates obtained at the Cape with the 24 -inch refractor.

(v) The series of 60 plates obtained with the Yale photographic refractor in Johannesburg.

(vi) The series of I4I plates obtained with the Franklin Adams telescope at the Union Observatory, Johannesburg.

(vii) The series of $4 \mathrm{I}$ plates obtained at the Melbourne Observatory with the I3-inch refractor. 
The reductions of series (i) to (v), based on the definitive star-places, have been completed; those of series (vi) and (vii) are in progress.

Bergedorf. Io plates obtained with the A.G. Astrograph, II plates obtained with the $60-\mathrm{cm}$. refractor, and 22 plates obtained with the Lippert Astrograph have been measured. The reductions, based on the definitive star-places, are expected to be completed by the end of 1938 .

Leipzig. The series of 32 plates, obtained with the $30-\mathrm{cm}$. refractor, has been measured and the reductions have been completed, provisional places for the comparison stars being used. The results are given in Veröff. der Univ. zu Leipzig, Heft 5 . Corrections to reduce to the definitive star-positions are being determined.

Uccle. The results of the measures of the series of 45 plates obtained with the Zeiss astrographic triplet were published in Bull. Astron. de l'Obs. roy. de Belgique, 2, No. 7. These results were based on the positions of the primary reference stars given in Kopff's catalogue. The corrected positions of Eros, based on the final positions of the reference stars, have been published in Bull. Astron. de l'Obs. roy. de Belgique, 2, No. Io. The 9 plates obtained with the astrographic refractor have been measured and reduced and the results have been communicated in manuscript.

Algiers. The results of the measures of II9 plates were published in $J . d . O . \mathbf{I}_{5}$, No. 9 and 17, No. 6. These were referred to the Kopff positions of the primary reference stars.

Catania. The series of 86 plates has been partly measured and reduced. I45 positions of Eros have been published in Mem. della Soc. Ast. Italiana, 9, No. I45. The measurement of the remaining plates (I29 positions of Eros) and the reduction of the whole material to the basis of the definitive star-positions will be completed in about $\mathrm{I} 8$ months.

Prague. I6 plates obtained with the astrographic refractor have been measured and reduced and the results have been published in Pub. de l'Obs. Nat. de Prague, No. 9, 1935. The results will be reduced shortly to the basis of the definitive star-places.

Pulkovo. The results of the measures of 20 plates obtained with the Normal Astrograph have been published in Pulk. Obs. Circ. No. 6, I933. Revised positions of Eros, based on the final star-places, are being computed.

Allegheny. The measurement of the important series of 146 plates obtained with the long-focus refractor is nearly completed. It is hoped that the reductions will be completed and the final positions of Eros will be available within a year.

Dearborn. 246 plates, with 2200 images of Eros, have been measured. Provisional reductions of some of these have been made. Now that the definitive secondary comparison star-places are available, the final reductions will be undertaken.

Lick. The measurement of the Eros plates is well advanced.

Washington. 50 plates taken with the I2-inch refractor have been measured; both primary and secondary reference stars appearing on the plates were measured. The reductions are now being undertaken, and it is hoped that final results will be available in about one year.

van Vleck. I22 plates, mostly with 8 exposures on Eros and 2 on the comparison stars, obtained with the long-focus refractor, have been measured. It is hoped to have the reductions completed by the middle of 1939 .

$Z \hat{o}-S e ̀$. The measures of 40 plates with the long-focus refractor were published in Annales de $Z \hat{o}-S e ̀$, I7, fasc. 6, I932. It is expected that the positions of Eros, based on the definitive catalogue positions will be available by the end of 1938 .

McCormick. 30 plates obtained with the long-focus refractor have been measured and reduced, using the Bergedorf places for the secondary reference stars. The 
results have been published in $A . J .46,172$, I937. The results of these plates and of 4 additional plates will be referred to the definitive star-places and published shortly.

Hyderabad. 2I2 plates were obtained with the astrographic refractor. The measurements have been nearly completed. The reductions will be based on the definitive star-places, and it is hoped that the final results will be ready for publication early in I939.

Cape. 532 plates obtained with the astrographic refractor have been measured and reduced, using Kopff's positions for the primary reference stars. The reduction to the definitive star-places will be completed at an early date.

La Plata. The measurement of the meridian series of 50 plates has been completed. It is expected that the definitive positions of Eros will be available within a year.

No report has been received from the following observatories: San Fernando, Tokyo, Lembang, Córdoba.

(b) Micrometric Observations.

Berlin-Babelsberg. The results of the observations made with the $65-\mathrm{cm}$. refractor at the I930-3I opposition are available in manuscript and will be published along with the observations at the $1937-38$ opposition. The results are expressed in the form $\xi=\left(\alpha_{\mathrm{E}}-\alpha_{*}\right) \cos \delta_{*} ; \eta=\delta_{\mathrm{E}}-\delta_{*}$ so that the positions of Eros, relative to the final positions of the comparison stars, are immediately available.

Pulkovo. The results of the observations with the I5-inch refractor, reduced with preliminary comparison star-places, have been published in Pulk. Obs. Circ. No. 4 .

Heidelberg. The observations on 15 nights with the I2-inch refractor have been reduced, with preliminary star-places, and the results have been communicated in manuscript.

Besançon. The results of 15 sets of observations, reduced to definitive positions, have been forwarded in manuscript.

Arcetri. The results of 32 observations of Eros have been published in the form $\Delta \alpha \cos \delta, \Delta \delta$ in Pub. R. Oss. Ast. Arcetri, No. 53 .

Padova. II8 groups were observed. The results have been published in the form $\Delta \alpha, \Delta \delta$ in R. Oss. Ast. Padova, Paper No. 34. The positions of Eros, based on the final star-places, are being derived.

Milano. The position remains as in the previous report.

Washington. It is anticipated that definitive positions of Eros, from the observations with the large visual refractor on 48 nights, will be available by the end of the year.

Teramo. The reductions of the micrometric observations will be completed and the definitive positions of Eros will be published during the present year.

Trieste. The results of the micrometric observations, in the form $\Delta \alpha, \Delta \delta$ were published in Mem. della Soc. Astron. Italiana, 9 (1935-36). The definitive positions of Eros from these and from some 30 transit circle observations will be available towards the end of the present year.

Wien. The final results of the micrometric observations will be published during the present year.

Union Observatory. The results of the visual observations with the long-focus refractor were published in U.O.C. No. 88, 1932 .

La Plata. The results of the micrometric observations were published in $A$ st. 
Nach. No. 6069. The positions of Eros, corrected to the definitive places of the reference stars have been communicated in manuscript.

No reports have been received from Kwasan and Lisbon.

3. The Path of Eros. The investigation of the path of Eros has been continued by $\mathrm{Dr}$ Witt, with the assistance of $\mathrm{Dr}$ von Schelling. The computations of the Saturn perturbations have been completed. The development of the perturbation function was carried out by all three methods of Hansen, in order to provide a complete example of the methods for Dr von Schelling and an assistant, who are undertaking the Uranus and Neptune perturbations. The computation of the Jupiter perturbations has also been completed. The Mars perturbations are being investigated; though these are not large numerically the number of terms to be taken into account is considerable.

It is to be anticipated that the investigation of the dynamical perturbations will lead to a value of the solar parallax equal to, or superior to, the value to be derived from the comparison of evening and morning observations at the same observatory or from practically simultaneous observations at different observatories. The solar parallax determined in the latter way may conceivably be systematically affected to a small extent by differential atmospheric dispersion. It is consequently important that the value so derived should be controlled by the value derived by an entirely different method.

4. The publication of the catalogue of positions of the secondary reference stars and of corrections to the positions of the primary reference stars, as given in Prof. Kopff's catalogue, marks an important stage in the investigation. It is hoped that all observatories that co-operated in securing observations at the opposition of r930-3I will complete the reductions and furnish the definitive positions of Eros as soon as possible. The Royal Observatory, Greenwich, has measured several series of plates taken at other observatories; if any observatory has a series of photographs that it cannot undertake to measure and reduce in the near future, it may be possible for the Greenwich Observatory to undertake the measurement and reduction.

An endeavour will be made to complete the discussion of the observational material, for the determination of the exact path of Eros, the solar parallax and the mass of the Moon before the meeting of the Union in I94I. The determination of the path is a necessary preliminary to the investigation of the solar parallax by the method of dynamical perturbations.

\author{
H. SPENCER JONES \\ President of the Sub-Commission \\ A. O. LEUSCHNER \\ President of the Commission
}

May I3, 1938 